The University of San Francisco

USF Scholarship: a digital repository @ Gleeson Library |

Geschke Center

2006

\title{
A Cosmic Milestone: Constraints from Metal-Poor Halo Stars on the Cosmological Reionization Epoch
}

Aparna Venkatesan

University of San Francisco, avenkatesan@usfca.edu

Follow this and additional works at: http://repository.usfca.edu/phys

Part of the Astrophysics and Astronomy Commons, and the Physics Commons

\section{Recommended Citation}

Aparna Venkatesan. A cosmic milestone: Constraints from metal-poor halo stars on the cosmological reionization epoch. 2006 Astrophysical Journal. 641 L81 doi:10.1086/504037

This Article is brought to you for free and open access by the College of Arts and Sciences at USF Scholarship: a digital repository @ Gleeson Library | Geschke Center. It has been accepted for inclusion in Physics and Astronomy by an authorized administrator of USF Scholarship: a digital repository @

Gleeson Library | Geschke Center. For more information, please contact repository@usfca.edu. 


\title{
A COSMIC MILESTONE: CONSTRAINTS FROM METAL-POOR HALO STARS ON THE COSMOLOGICAL REIONIZATION EPOCH
}

\author{
Aparna Venkatesan ${ }^{1}$ \\ Center for Astrophysics and Space Astronomy, Department of Astrophysical and Planetary Sciences, UCB 389, \\ University of Colorado, Boulder, CO 80309-0389; aparna@ casa.colorado.edu \\ Received 2005 December 30; accepted 2006 March 8; published 2006 April 3
}

\begin{abstract}
Theoretical studies and current observations of the high-redshift intergalactic medium (IGM) indicate that at least two cosmic transitions occur by the time the universe reaches gas metallicities of about $10^{-3} Z_{\odot}$. One is the cosmological reionization of the IGM, and the second is the transition from a primordial to present-day mode of star formation. We quantify this relation through new calculations of the ionizing radiation produced in association with the elements carbon, oxygen, and silicon observed in Galactic metal-poor halo stars, which are likely second-generation objects formed in the wake of primordial supernovae. We demonstrate that sufficient ionizing photons per baryon are created by enrichment levels of $[\mathrm{Fe} / \mathrm{H}] \sim-3$ in the environment of metal-poor halo stars that provide the optical depth in the cosmic microwave background of $\sim 0.1$ detected by WMAP. We show that, on a star-by-star basis, a genuine cosmic milestone in the ionization of the IGM and in the mode of star formation occurred at metallicities of $10^{-4}$ to $10^{-3} Z_{\odot}$ in these halo stars. This provides us with an important link in the chain of evidence for metal-free first stars having dominated the process of reionization by $z \sim 6$. We conclude that many of the Fe-poor halo stars formed close to the end of or soon after cosmological reionization, making them the ideal probe of the physical conditions under which the transition from first- to second-generation star formation happened in primordial galaxies.
\end{abstract}

Subject headings: cosmology: theory — galaxies: high-redshift nuclear reactions, nucleosynthesis, abundances — stars: abundances — stars: Population II

Online material: color figures

\section{INTRODUCTION}

Theoretical studies of the early universe predict the existence of first-generation stars that lack metals and consequently have unique physical structures and properties (Bromm et al. 2001b; Tumlinson et al. 2003; Schaerer 2002). Such objects, however, have not been detected to date in either the high-redshift or local universe. Therefore, indirect inferences from modeling the impact of primordial stars and supernovae $(\mathrm{SNe})$ on their environment through radiative and chemical feedback remain important. The reionization of the intergalactic medium (IGM) and the widespread metal enrichment of high- $z$ galaxies, QSOs, and the IGM provide us with strong observational constraints for such theoretical studies. A particularly effective tool in constraining the masses and properties of the first stars has been to combine their ionizing photon and nucleosynthetic signatures (Venkatesan \& Truran 2003; Tumlinson et al. 2004).

When we examine the current data and the results of theoretical work, an interesting coincidence becomes apparent: three important cosmic transitions occur at gas metallicities of about $10^{-5}$ to $10^{-3} Z_{\odot}$. To begin with, the universe first generates sufficient ionizing photons for hydrogen reionization when the IGM reaches these metallicities (Miralda-Escude \& Rees 1997; Venkatesan \& Truran 2003). Second, theoretical studies of the cooling and fragmentation of gas in early galaxies reveal that the primordial stellar initial mass function (IMF) evolves from its metal-free to presentday form at gas transition metallicities of $Z_{\mathrm{tr}} \sim 10^{-5}$ to $10^{-3} Z_{\odot}$ (Bromm et al. 2001a; Schneider et al. 2002; Omukai et al. 2005; Santoro \& Shull 2006). This transition results from the physics of gas cooling in early galaxies, when the dominant cooling channel switches from molecular hydrogen to metal lines or dust grains, with the latter pathway dominating in systems with

${ }^{1}$ NSF Astronomy and Astrophysics Postdoctoral Fellow.
$Z \lesssim 10^{-4} Z_{\odot}$ (Omukai et al. 2005). Third, there are indications that the relative abundances of $\mathrm{CNO}$ and $\alpha$-elements in metalpoor stars in the Galactic halo have increased scatter and altered ratios when the iron content of these stars falls below $\sim 10^{-3}$ of solar values (Barklem et al. 2005; Cayrel et al. 2004; Beers \& Christlieb 2005 and references therein). These subsolar mass objects are thought to be second-generation stars that formed from metal-poor fragmenting gas in the shells of the very first $\mathrm{SNe}$ in early galaxies (Salvaterra et al. 2004). Such stars contain the fossil imprint of nucleosynthesis from the preceding generations of metal-free stars, reflecting the "prompt inventory" of metals (Qian \& Wasserburg 2002; Wada \& Venkatesan 2003).

When these three points are taken together, we see that several cosmological milestones occurred by metallicities of $\sim 10^{-3} Z_{\odot}$, a relation that must result from the strong feedback between the buildup of ionizing radiation and the formation of second-generation stars. In this Letter, we quantitatively demonstrate a novel connection in the environment of extremely metal-poor (EMP) halo stars between two of these relationships; i.e., the stellar population that was important for early nucleosynthesis is also responsible for cosmological reionization. We define EMP stars as those with $[\mathrm{Fe} / \mathrm{H}] \lessgtr-3$ (Beers $\&$ Christlieb 2005). We examine the significance of the second transition in relation to the other two in a subsequent work, where we plan to use numerical simulations to investigate the spatially varying feedback between gas cooling and the propagation of ionizing radiation in the first galaxies.

\section{STELLAR ABUNDANCES AND IONIZING EFFICIENCY}

We begin by showing the trends of element abundances in EMP stars from current observations. We follow our approach in Venkatesan et al. (2006) and take the data from Cayrel et al. (2004) and Tumlinson et al. (2004 and references therein). 
For the two most iron-poor stars, HE 0107-5240 and HE 1327-2326, we use the published abundances from Christlieb et al. (2004), Aoki et al. (2004), and Frebel et al. (2005, 2006). The last of these papers has recently revised the CNO abundances of HE 1327-2326 to take into consideration threedimensional non-LTE corrections. We focus on the elements $\mathrm{C}, \mathrm{O}, \mathrm{Si}$ and $\mathrm{Fe}$, as these are among the most dominant metals created in primordial $\mathrm{SNe}$ and are the most important coolants in metal-free gas (Bromm \& Loeb 2003; Santoro \& Shull 2006).

The trends of $[\mathrm{C} / \mathrm{H}],[\mathrm{O} / \mathrm{H}],[\mathrm{Si} / \mathrm{H}]$, and $[\mathrm{C} / \mathrm{O}]$ as a function of iron abundance $[\mathrm{Fe} / \mathrm{H}]$ are shown in Figure 1, where for an element $\mathrm{X}$, we define $[\mathrm{X} / \mathrm{H}]$ as the ratio of the measured column densities of $\mathrm{X} / \mathrm{H}$ to its solar ratio. We display those points with $[\mathrm{Fe} / \mathrm{H}] \lesssim-2$ in order to span the predicted $Z_{\mathrm{tr}}$ in individual elements (Bromm \& Loeb 2003; Santoro \& Shull 2006), as we will see below. We assume that EMP stars with $[\mathrm{Fe} / \mathrm{H}] \lesssim$ -3 have been enriched only by metal-free stars, consistent with the estimate of $Z_{\mathrm{tr}}$ from Santoro \& Shull (2006) and with recent calculations using merger tree models in which stars of metallicities $\leqslant 10^{-3} Z_{\odot}$ reflect enrichment from only one to a few primordial SNe (Tumlinson 2006).

Figure 1 reveals some interesting trends. First, for those stars that have both abundances measured, [C/O] shows no clear trend with $[\mathrm{Fe} / \mathrm{H}]$; in fact, it appears to have its maximum value at the lowest $[\mathrm{Fe} / \mathrm{H}]$. Such element abundance ratios are often used as a cosmic clock to track the creation of elements from stars of differing lifetimes, but such a relation may not be easily derived in EMP stars, a point that we return to in the discussion. Second, carbon and silicon roughly track Fe in enrichment relative to $\mathrm{H}$, whereas oxygen does not. Again, the exceptions to this trend are the most Fe-poor stars. Note that some of the stellar data shown here come from surveys that select against C-rich EMP stars (Cayrel et al. 2004), owing to the complicated analyses involved in such cases. The more recent survey by Barklem et al. (2005) does not have this bias, although these authors focus mostly on $r$-process element abundances in EMP stars.

A direct translation of the observed abundances in EMP halo stars to yields from primordial SNe and constraints on the first stars' IMF would be ideal, but it is complicated by several factors. Mass transfer from a companion may alter original abundance ratios, but it is difficult to model owing to the unknown binary fraction of the first stellar generations and the role of asymptotic giant branch phase dredge-ups and mass loss in metal-free stars. We have not plotted those EMP stars known to be in binaries in Figure 1, but it is possible that many of these stars have as yet undetected companions. A uniform sample coverage of the $\mathrm{CNO}$ elements would constrain this scenario better.

In order to assess the implications of the nucleosynthetic data in EMP stars for reionization, we follow the approach in Madau \& Shull (1996) and Venkatesan \& Truran (2003). We calculate the ionizing efficiency, $\eta_{\text {Lyc }}$, which is essentially a conversion factor defined as the ratio of the energy produced in rest mass of metals, $M_{Z} c^{2}$, to the energy released in the $\mathrm{H}-$ ionizing continuum:

$$
\eta_{\text {Lyc }} \equiv E(h \nu \geq 13.6 \mathrm{eV}) /\left(M_{Z} c^{2}\right) .
$$

The first stars are expected to be metal-free in composition, a factor that critically influences their structure and leads to the emission of significantly harder ionizing radiation relative to low- $Z$ stars (Bromm et al. 2001b; Tumlinson et al. 2003;

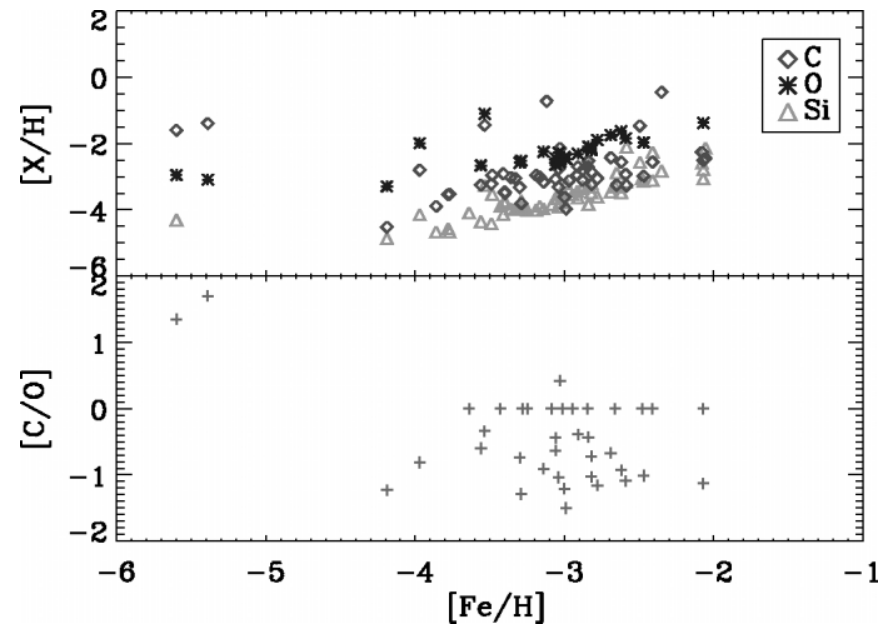

FIG. 1.-Current data from Galactic metal-poor halo stars on the abundances of $\mathrm{C}, \mathrm{O}$, and $\mathrm{Si}$ relative to $\mathrm{H}$, and of $[\mathrm{C} / \mathrm{O}]$, as a function of $[\mathrm{Fe} / \mathrm{H}]$. [See the electronic edition of the Journal for a color version of this figure.]

Schaerer 2002). Venkatesan \& Truran (2003) showed that $\eta_{\text {Lyc }}$ is a strong function of stellar metallicity and increases by a factor of $10-20$ from $Z=Z_{\odot}$ to $Z=0$ stars, owing to the boosted ionizing flux and substantially reduced metal yield from massive stars with decreasing stellar metallicity. The latter trend is a result of the more compact structure of metal-poor stars, which directly leads to greater element fallback onto the remnant after the $\mathrm{SN}$ explosion.

The stellar IMF is assumed to have the form $\phi(M)=$ $\phi_{0} M^{-\alpha}$, which is normalized over the mass range as $\int d M M \phi(M)=1$. We consider two primordial IMFs, one that has $100-1000 M_{\odot}$ stars with a flat IMF slope (hereafter very massive stars [VMSs]), as some theoretical studies indicate (Omukai \& Palla 2003; Bromm et al. 2002; Nakamura \& Umemura 2002), and a present-day IMF with $1-100 M_{\odot}$ stars where the IMF slope has a Salpeter value, $\alpha=2.35$. We use the ionizing spectra from Bromm et al. (2001b) and Schaerer (2002) for VMSs, and the results from Tumlinson et al. (2003) for the 1-100 $M_{\odot}$ IMF. We take the metal yields from Woosley \& Weaver (1995) for 10-40 $M_{\odot}$ stars, which end their lives as Type II SNe, and from Heger \& Woosley (2002) for stars with masses $140-260 M_{\odot}$ that explode entirely as pair-instability SNe. All other massive stars are assumed to collapse into black holes with no contribution to the nucleosynthetic output. Varying the upper and lower mass limits in the VMS IMF will not significantly impact our conclusions, owing to the narrow mass range of the element synthesis from VMS SNe and to the near-identical ionizing photon production per stellar baryon in all stars with masses $\gtrsim 300 M_{\odot}$ (Bromm et al. 2001b).

We do not include cases in which the conversion efficiency is derived by including the metal yield from intermediate-mass stars, as $\eta_{\text {Lyc }}$ remains approximately the same for oxygen and exactly the same for $\mathrm{Si}$. For carbon, $\eta_{\mathrm{Lyc}}$ decreases by a factor of a few with intermediate-mass stars owing to their large yield in C. For the purposes of this Letter, we wish to track the growth of ionizing radiation that is dominated by the massive stars in the IMF. We do not include the yield from Type Ia $\mathrm{SNe}$ in our calculations. Recent studies indicate that such $\mathrm{SNe}$ may not occur in metal-free stellar populations (Kobayashi et al. 1998), although this is a sensitive function of the SN mechanism itself and of the unknown binarity in $Z=0$ stars.

As shown in Venkatesan \& Truran (2003) and MiraldaEscude \& Rees (1997), calculations of $\eta_{\text {Lyc }}$ can be used to com- 
pute the number of ionizing photons per baryon generated in association with a metallicity that is observed in a given system. These papers focused on the IGM, and they pointed out that the universe first generates about 10 ionizing photons per IGM baryon for the observed IGM metallicity of $\sim 10^{-2.5} Z_{\odot}$. This estimate, however, is subject to the uncertainties of star formation efficiency and the escape fraction of ionizing radiation from early galaxies. Here we examine the ionizing efficiency in the environment of EMPs and calculate $N_{\gamma} / N_{b}$, the number of ionizing photons created per baryon in stars, which is not related to the above, highly uncertain astrophysical parameters. Following Venkatesan \& Truran (2003), for an element $i, N_{\gamma, i} / N_{b}=$ $\eta_{\text {Lyc, } i} Z_{i}\left(1 \mathrm{GeV} /\left\langle E_{\text {Lyc }}\right\rangle\right)$; we assume the IMF-averaged energy of a Lyman continuum photon, $\left\langle E_{\mathrm{Lyc}}\right\rangle=27 \mathrm{eV}(30 \mathrm{eV})$ for metalfree stars in a present-day (VMS) IMF.

\section{RESULTS AND DISCUSSION}

For our calculations here, we take the values of $\eta_{\mathrm{Lyc}}$ for carbon and oxygen computed in Venkatesan \& Truran (2003), and we compute them independently for silicon. We find that for the $1-100 M_{\odot}$ Salpeter-slope IMF, $\eta_{\mathrm{Lyc}}$ has values of 0.48 , 0.2 , and 1.34 for $\mathrm{C}, \mathrm{O}$, and $\mathrm{Si}$, respectively; for a VMS IMF, the corresponding values are 0.098, 0.01, and 0.057. Using these numbers, we show the results of our calculations in Figure 2 , where the number of ionizing photons created per baryon in EMP stars, $N_{\gamma} / N_{b}$, is shown as a function of $[\mathrm{Fe} / \mathrm{H}]$ for the elements $\mathrm{C}, \mathrm{O}$, and $\mathrm{Si}$ in three separate panels. In each case, the ionizing photon contribution from a present-day IMF and a VMS IMF are plotted.

A number of trends seen in Figure 2 are of relevance to the first stars' field. First, VMSs make a low but persistent contribution to the overall ionizing photon budget in association with the metals locked in EMP stars, typically an order of magnitude less than the $1-100 M_{\odot}$ IMF. This is because VMSs are in general less efficient at generating total ionizing radiation per unit metal yield relative to other metal-free stellar populations, owing to the large metal production from VMSs. Second, the slow buildup of metals and the associated ionizing radiation are apparent, for each element and for each IMF. This trend appears most obvious for oxygen, although there is a significant scatter in the correlation between $N_{\gamma} / N_{b}$ and $[\mathrm{Fe} / \mathrm{H}]$ at any given $[\mathrm{Fe} / \mathrm{H}]$. As we stated earlier, the notable exceptions are the two stars at the lowest values of $[\mathrm{Fe} / \mathrm{H}]$.

Third, we show in each panel the approximate criterion to generate an optical depth in the cosmic microwave background (CMB) of about 0.1, consistent with data from the Wilkinson Microwave Anisotropy Probe (WMAP; Spergel et al. 2006), of about $N_{\gamma} / N_{b} \sim 34,000$ (Tumlinson 2006; Tumlinson et al. 2004). This number is derived from interpolating between cases studied by Tumlinson et al. (2004) in which the lifetime-integrated ionizing photon production from various stellar populations and IMFs were calculated and used as inputs in detailed cosmological reionization models. Interestingly, the EMP data indicate that this line is crossed at least once and perhaps twice in the range $-4 \leq[\mathrm{Fe} / \mathrm{H}] \leq-3$ by a present-day IMF. This is best seen in the panel corresponding to $\mathrm{Si}$, where a clear dip in $N_{\gamma} / N_{b}$ occurs in the range $-3.6 \leq[\mathrm{Fe} /$ $\mathrm{H}] \leq-3$. The VMS IMF appears to never meet this criterion over the metallicity ranges considered here, consistent with the role of VMSs in IGM reionization predicted by Venkatesan \& Truran (2003).

Conducting a metal census of the universe from EMP stars and from the IGM is important in both environments, despite their differing systematics. The IGM contains nearly all of the baryons

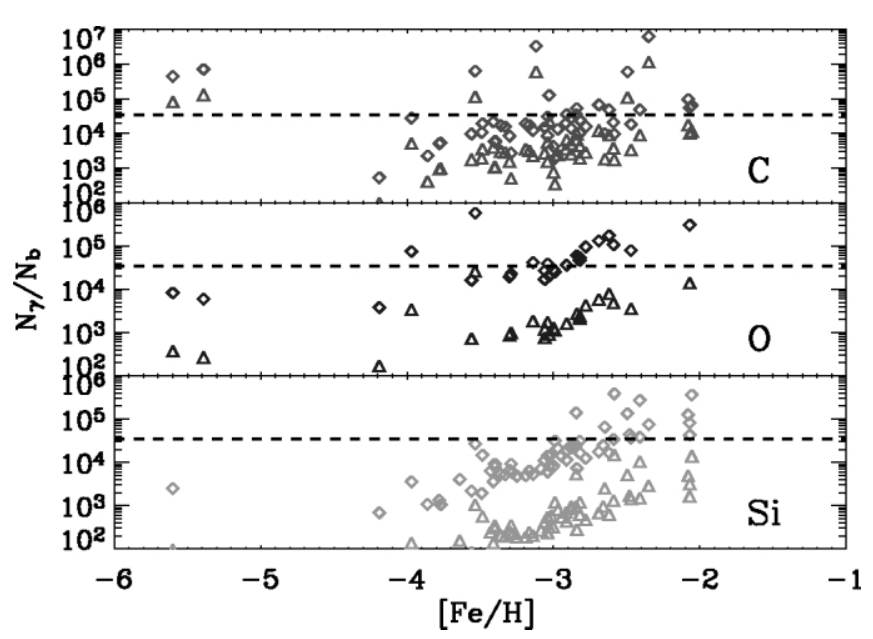

FIG. 2.-Minimum number of ionizing photons per baryon in stars that is created in association with specific metals observed in EMP halo stars. The top, middle, and bottom panels correspond to the elements $\mathrm{C}, \mathrm{O}$, and $\mathrm{Si}$, respectively. The thick dashed black line in each panel corresponds to the value required to roughly match the WMAP data for an optical depth of $10 \%$ in the CMB. All cases are for metal-free stars; each panel displays values for the 1-100 $M_{\odot}$ (diamonds) and VMS (triangles) IMFs considered in this work. See text for discussion. [See the electronic edition of the Journal for a color version of this figure.]

at high redshifts, but the former case permits us to undertake a detailed star-by-star analysis of the cosmic-enrichment history. By working with nucleosynthetic data in EMP stars rather than the IGM, we have not assigned a global fixed metallicity with the appropriately scaled individual element values according to their solar ratios. Rather, we have derived parallel constraints for reionization from a number of elements independently, which is a considerably stronger approach. In addition, observations of EMP stars relative to IGM absorbers provide significantly larger element coverage in the well-constrained environment of stellar atmospheres, a situation that will greatly improve in the near future.

Although we naively apply the same factor for ionization efficiency for $[\mathrm{Fe} / \mathrm{H}] \gtrsim-3, \eta_{\mathrm{Lyc}}$ is likely to be closer to the values associated with metal-poor stars, typically a factor of a few to 10 lower, owing to their increased metal yield. Depending on the individual metal abundance, this could lead to a less steep rise or flattening in the behavior of $N_{\gamma} / N_{b}$ at $[\mathrm{Fe} / \mathrm{H}] \gtrsim-3$. This prediction is somewhat more speculative, as the integrated contribution from many subsequent stellar generations of varying metallicities will be difficult to distinguish in this environment.

The notable exceptions to most of the trends noted above are the two most $\mathrm{Fe}$-poor stars at $[\mathrm{Fe} / \mathrm{H}] \sim-5.5$. At such low metallicities, one would expect that these stars reflect enrichment from a single SN (Tumlinson 2006). These stars, however, are highly overenriched in several elements including $\mathrm{C}, \mathrm{O}$, and $\mathrm{Si}$, to the degree that they are actually not metal-poor but rather at metallicities of nearly a tenth solar. How these stars came to acquire such skewed element abundances relative to solar ratios remains puzzling at present. Some possibilities include pollution from a binary companion, pollution from a subluminous hypernova with fine-tuned ratios of mixing and fallback (Iwamoto et al. 2005), or the effects of stellar rotation and ensuing mass loss that could dramatically boost the production of CNO elements (Chiappini et al. 2006). The ratio of these elements to iron or relative to each other is sometimes used as a chronometer in other astrophysical environments. For the most $\mathrm{Fe}$-poor stars, however, it is clear that ratios such as $[\mathrm{Fe} / \mathrm{O}]$ or $[\mathrm{C} / \mathrm{O}]$ are not easily related to time. These should 
normally increase with the age of a stellar population as the stars of progressively lower mass evolve off the main sequence, but as we see in Figure 1 for EMP stars, [C/O] achieves its highest values at the lowest $[\mathrm{Fe} / \mathrm{H}]$. Perhaps these stars were created in atypical environments as the IGM approached reionization, as seen from the generation of ionizing radiation in Figure 2-the most Fe-poor stars appear to have formed at epochs when, for a metal-free present-day IMF, $N_{\gamma} / N_{b}$ nearly equals or exceeds the criterion from WMAP, at least for $\mathrm{C}$. One interesting explanation for this, in addition to those above, could be that the most Fe-poor halo stars are also true secondgeneration stars, ones that formed through the dust grain cooling pathway $\left(Z_{\mathrm{tr}} \sim 10^{-5} Z_{\odot}\right)$ rather than through metal line cooling using $\mathrm{C}, \mathrm{O}$, or $\mathrm{Si}\left(Z_{\mathrm{tr}} \sim 10^{-3} Z_{\odot}\right)$. The unusual element trends in these stars could then be explained through the element segregation predicted in a scenario involving dust transport in early galaxies. Here conditions in primordial SN remnants result in the radiative transport of dust in the hot radiation field from metal-free stars, leading to selective element enhancement from compounds such as graphites and silicates in the cooling gas in SN shells (Venkatesan et al. 2006), which are likely the sites of EMP star formation.

What do the results in this Letter imply for cosmology? Those EMP stars that have observational age estimates are about 12-13 Gyr old (Sneden et al. 2003; Beers \& Christlieb 2005). In the context of our results, it appears that most of the EMP stars formed close to the end of or soon after cosmological reionization. That reionization occurs in the first gigayear of the universe (by $z \sim 6$ ) is no surprise, as this is already clear from Gunn-Peterson studies of the high- $z$ IGM (Becker et al. 2001) and from current CMB data. A more interesting result of translating the EMP star abundances in $\mathrm{C}, \mathrm{O}$, and $\mathrm{Si}$ into the accompanying production of ionizing photons per baryon in stars is the clear buildup of ionizing radiation until reionization is accomplished at $[\mathrm{Fe} / \mathrm{H}] \sim-3,[\mathrm{Si} / \mathrm{H}] \sim-3.5$, and $[\mathrm{O} / \mathrm{H}] \sim-2.5([\mathrm{C} / \mathrm{H}]$ ranges from -4 to -2 at these Fe metallicities). At this point, the initial stellar population (presumably metal-free) must turn off, as seen from the altered element abundance patterns at higher $[\mathrm{Fe} / \mathrm{H}]$, leading to a new pattern of nucleosynthesis from different SNe. Cayrel et al. (2004) argue for the presence of a primordial SN population up to $[\mathrm{Fe} / \mathrm{H}]$ or $[\mathrm{Mg} / \mathrm{H}]$ values of about -3 , with a combination of primordial and nonprimordial $\mathrm{SNe}$ at higher metallicities. Such a truncation in the mode of star formation is most likely attributed to the critical metallicity being reached in the gas in which EMP stars formed, signaling an end to the first stars' epoch. Given certain conditions of density and temperature, $Z_{\text {tr }}$ can be higher in iron than in carbon, oxygen, or silicon. This is not consistent, however, with the overall trend of enhanced $\mathrm{C}, \mathrm{O}$, and $\mathrm{Si}$ at low $[\mathrm{Fe} / \mathrm{H}]$. A naive projection of the above metallicities in $\mathrm{Fe}, \mathrm{Si}, \mathrm{O}$, and $\mathrm{C}$ at which reionization "occurs" in Figure 2 implies that EMP stars should have formed in gas of number densities $\gtrsim 10^{3} \mathrm{~cm}^{-3}$ (Santoro \& Shull 2006).

A more puzzling coincidence is why the end of the first stars' phase should occur at about the epochs corresponding to cosmological reionization. This points toward the possibility of strong feedback between the intrahalo metal reincorporation timescales and the halo-IGM transport of ionizing radiation. We return to this issue in a subsequent work, where we will quantitatively relate the reionization epoch to the transition metallicity in early galaxies. Clearly, EMP stars provide an important window to study the transition from first- to second-generation star formation and the physical conditions at which this occurs in primordial galaxies. The novel result derived here is that the abundances in such stars show a strong relation to cosmological reionization. Targeted observations are required with more uniform element coverage of EMP stars, particularly the C-rich stars, in the critical range of $-6 \lesssim[\mathrm{Fe} / \mathrm{H}] \lesssim-4$. This range currently has very little data and will be useful in closing the loop in our understanding of these important cosmic events at high redshifts.

I thank Tim Beers, Jason Tumlinson, and an anonymous referee for helpful comments on the manuscript. I gratefully acknowledge the support of NSF grant AST-0201670 through the NSF Astronomy and Astrophysics Postdoctoral Fellowship program.

\section{REFERENCES}

Aoki, W., Norris, J. E., Ryan, S. G., Beers, T. C., Christlieb, N., Tsangarides, S., \& Ando, H. 2004, ApJ, 608, 971

Barklem, P. S., et al. 2005, A\&A, 439, 129

Becker, R. H., et al. 2001, AJ, 122, 2850

Beers, T., \& Christlieb, N. 2005, ARA\&A, 43, 531

Bromm, V., Coppi, P. S., \& Larson, R. B. 2002, ApJ, 564, 23

Bromm, V., Ferrara, A., Coppi, P. S., \& Larson, R. B. 2001a, MNRAS, 328, 969

Bromm, V., Kudritzki, R. P., \& Loeb, A. 2001b, ApJ, 552, 464

Bromm, V., \& Loeb, A. 2003, Nature, 425, 812

Cayrel, R., et al. 2004, A\&A, 416, 1117

Chiappini, C., Hirschi, R., Meynet, G., Ekström, S., Maeder, A., \& Matteucci, F. 2006, A\&A, in press (astro-ph/0602459)

Christlieb, N., Gustafsson, B., Korn, A. J., Barklem, P. S., Beers, T. C., Bessell, M. S., Karlsson, T., \& Mizuno-Wiedner, M. 2004, ApJ, 603, 708

Frebel, A., Christlieb, N., Norris, J. E., Aoki, W., \& Asplund, M. 2006, ApJ, 638, L17

Frebel, A., et al. 2005, Nature, 434, 871

Heger, A., \& Woosley, S. E. 2002, ApJ, 567, 532

Iwamoto, N., Umeda, H., Tominaga, N., Nomoto, K., \& Maeda, K. 2005, Science, 309, 451
Kobayashi, C., Tsujimoto, T., Nomoto, K., Hachisu, I., \& Kato, M. 1998, ApJ, 503, L155

Madau, P., \& Shull, J. M. 1996, ApJ, 457, 551

Miralda-Escude, J., \& Rees, M. J. 1997, ApJ, 478, L57

Nakamura, F., \& Umemura, M. 2002, ApJ, 569, 549

Omukai, K., \& Palla, F. 2003, ApJ, 589, 677

Omukai, K., Tsuribe, T., Schneider, R., \& Ferrara, A. 2005, ApJ, 626, 627

Qian, Y.-Z., \& Wasserburg, G. J. 2002, ApJ, 567, 515

Salvaterra, R., Ferrara, A., \& Schneider, R. 2004, NewA, 10, 113

Santoro, F., \& Shull, J. M. 2006, ApJ, in press (astro-ph/0509101)

Schaerer, D. 2002, A\&A, 382, 28

Schneider, R., Ferrara, A., Natarajan, P., \& Omukai, K. 2002, ApJ, 571, 30

Sneden, C., et al. 2003, ApJ, 591, 936

Spergel, D. N., et al. 2006, ApJ, submitted (astro-ph/0603449)

Tumlinson, J. 2006, ApJ, in press (astro-ph/0507442)

Tumlinson, J., Shull, J. M., \& Venkatesan, A. 2003, ApJ, 584, 608

Tumlinson, J., Venkatesan, A., \& Shull, J. M. 2004, ApJ, 612, 602

Venkatesan, A., Nath, B. B., \& Shull, J. M. 2006, ApJ, 640, 31

Venkatesan, A., \& Truran, J. W. 2003, ApJ, 594, L1

Wada, K., \& Venkatesan, A. 2003, ApJ, 591, 38

Woosley, S. E., \& Weaver, T. A. 1995, ApJS, 101, 181 